\title{
La transition entre vapeur et électricité Exemples vécus (Mohon, Strasbourg, Noisy-le-Sec)
}

The transition between steam and electric energy. Real-life examples (Mohon, Strasbourg, Noisy-le-Sec)

André Blanc

\section{OpenEdition}

\section{Journals}

Édition électronique

URL : https://journals.openedition.org/rhcf/1780

DOI : 10.4000/rhcf. 1780

Éditeur

Rails \& histoire

Édition imprimée

Date de publication : 1 décembre 2003

Pagination : 286-319

ISBN : 0996-9403

ISSN : 0996-9403

Référence électronique

André Blanc, "La transition entre vapeur et électricité Exemples vécus (Mohon, Strasbourg, Noisy-leSec) », Revue d'histoire des chemins de fer [En ligne], 28-29 | 2003, mis en ligne le 30 décembre 2014, consulté le 22 avril 2022. URL : http://journals.openedition.org/rhcf/1780 ; DOI : https://doi.org/ $10.4000 /$ rhcf. 1780 


\section{La transition entre vapeur et électricité Exemples vécus (Mohon, Strasbourg, Noisy-le-Sec)}

Je voudrais modestement évoquer par quelques souvenirs la vie quotidienne dans certains dépôts de l'Est au moment de cette profonde mutation : le passage de la traction à vapeur à la traction électrique ou thermique, c'est-à-dire entre 1950 et 1960.

\section{Rappelons tout d'abord le cadre}

Beaucoup de dépôts avaient été sévèrement bombardés, ou leurs installations sabotées lors de la retraite allemande ; les premiers efforts de l'immédiat après-guerre (par exemple des machines-outils protégées de la pluie et de la neige par des bâches) ont été déployés pour obtenir une remise en service du chemin de fer la plus rapide possible, des logements détruits ont été remplacés par des baraquements en bois. Puis la reconstruction a permis progressivement de disposer d'installations mieux adaptées (rotondes type P ou remises rectangulaires), des dépôts reconstruits étaient plus à l'aise, déplacés au besoin : Châlons, Noisy, Chaumont-Chalindrey, Nancy, Metz, Thionville. Au début des années cinquante, l'essentiel venait d'être achevé.

\section{Les hommes}

D’une façon générale, l'encadrement était de qualité : ingénieurs de grandes écoles embauchés dans les années 1930-1940, expérimentés et des cadres de valeur, issus de la promotion interne ; puis des embauchages supérieurs aux besoins furent effectués pendant la guerre pour limiter les départs en Allemagne... mais bon nombre d'entre ceux qui, initialement, n'avaient pas prévu de faire carrière au chemin de fer furent séduits par l'intérêt et par l'activité du métier ; la conséquence en a été une situation assez figée dans les années 1950-1960. À titre d'exemple, mon successeur au dépôt de Noisy-le-Sec, en 1962, avait tenu pendant sept ans le poste de chef de feuille (c'est-à-dire la programmation du service des agents de conduite) au dépôt de Chalindrey ; bien que ce métier exige de solides qualités humaines, il n'était pas vraiment nécessaire d'être ingénieur des Arts et Métiers pour l'exercer!

Une autre conséquence fut une baisse très sensible du nombre d'embauches des jeunes ingénieurs pendant plusieurs années, accompagnée d'un niveau de rémunération qui s'écartait de plus en 
plus de ce qui était offert en général dans l'industrie. Plusieurs démissions se produisirent dans ces conditions sans qu'il y ait d'efforts pour les retenir, au contraire, d'autant que la sidérurgie, notamment, était intéressée par ces cadres encore jeunes et déjà expérimentés.

Le personnel d'entretien était essentiellement issu de l'apprentissage SNCF, qu'il s'agisse des compagnons ou de la maitrise ; c'était d'une façon très générale un personnel compétent et attaché au métier ; la notion de l'importance du transport ferroviaire dans l'activité nationale était encore très forte. Il ne faut en effet pas oublier que la grande majorité des agents de tous grades sortaient de la période de guerre encore très proche alors, où le transport ferroviaire était vital pour l'alimentation du pays et l'activité économique subsistante ; des trésors d'ingéniosité et de débrouillardise avaient été déployés pour répondre aux besoins du pays malgré les difficultés et les dangers de tous ordres, en cherchant à contourner la priorité officielle des transports militaires de l'occupant sur laquelle veillaient les fonctionnaires de la Deutsche Reichsbahn placés auprès des emplois «stratégiques ».

Une telle période avait laissé des traces profondes même si les intéressés n'en parlaient que rarement, peut-être pour tenter d'effacer les mauvais souvenirs.

Pour le personnel de conduite, les mécaniciens venaient également de l'apprentissage, mais les chauffeurs comportaient une part appréciable d'agents à l'origine manœuvres, du fait des difficultés de recrutement face aux besoins de la reconstruction et de la durée limitée dans ces emplois des ex-apprentis. En complément des formations dispensées par les chefs mécaniciens (devenus ensuite chefs de Traction), la pratique du métier était pour une large part acquise par compagnonnage : le mécanicien formait son chauffeur puis le préparait à l'examen d'élèvemécanicien, en liaison avec la maitrise. À l'atelier, c'était le rôle des chefs d'équipe et des contremaitres. J'ai parfois entendu critiquer les principes du compagnonnage, l'apprenti étant trop soumis au bon vouloir de son « chef». Bien sûr, j’ai connu quelques cas de l'espèce ; mais je dois dire que dans les dépôts de l'Est où j’ai été en poste, il ne s'agissait que de cas exceptionnels ; dans l'ensemble, les mécaniciens ou les dirigeants d'atelier à qui on confiait les débutants avaient un profond souci d'enseigner le métier (et ses " ficelles ») même si les méthodes étaient parfois un peu rugueuses. Les conversations que j’ai eues par la suite m’ont confirmé dans cette impression : les «bras d'acier » étaient bien connus et n'étaient pas les plus estimés de leurs collègues!

Souvent, c'était le mariage qui avait orienté les jeunes vers la sédentarité de l'atelier ou les horaires bien variables des services de 
conduite ; encore faut-il noter que les ouvriers devaient assurer des renforts dans les rangs des chauffeurs pendant les pointes de trafic, parfois à contre-cœur!

Surtout dans les dépôts chargés de locomotives à vapeur, cette homogénéité de l'origine du personnel n'était pas sans importance.

En effet, plus que les ateliers, les dépôts sont marqués par le quotidien et ses aléas : il faut « sortir » les machines à l'heure pour assurer les trains et l'engagement du personnel conditionne largement la réussite, surtout en traction vapeur où les imprévus étaient plus fréquents. Depuis des décennies, la liaison entre les agents de conduite et ceux chargés de l'entretien était constante et nécessaire pour orienter au mieux les interventions de la vie courante ${ }^{1}$. Cette liaison est évidemment facilitée par une formation commune, donc un langage commun. La fiabilité des engins moteurs récents et le regroupement des centres d'entretien a considérablement réduit la fréquence de ces contacts sans toutefois en supprimer la nécessité ; mais leur forme a changé.

Avec la traction électrique ou Diesel, le rôle du deuxième agent de conduite s'est rapidement amenuisé au cours de la période considérée ; il a quasiment disparu maintenant. La formation par contact direct s'est donc progressivement réduite, celle des ouvriers d'entretien est devenue plus méthodique, certains diraient plus professionnelle. Si la formation théorique a gagné en efficacité, elle a peut-être un peu perdu en relation humaine quotidienne entre le formé et le formateur et en expérience pratique des petits incidents.

Avec le regroupement des centres d'entretien, le contact direct entre conducteurs et ouvriers est devenu l'exception en service courant; il a été progressivement remplacé par des procédures de signalement. Remarquons qu'en ce domaine aussi les $141 \mathrm{R}$ ont, avec leur large banalisation, amorcé l'ère de la traction moderne.

On peut esquisser une image (bien sûr approximative) de cette évolution, en disant qu'en dehors des organes sensibles - les chaudières, les organes de roulement - dont l'entretien était déjà bien codifié, on est passé de l'artisanat à un entretien industriel programmé.

Comment, devant ces changements, le personnel a-t-il adapté son comportement en conservant la marge d'initiative personnelle qui lui est nécessaire?

1- Voir, in fine, le témoignage d'un ancien mécanicien, Daniel Lécheneau, qui évoque ces liaisons. 
Car je rappelle cette réflexion d'Alain : «L'homme n'est heureux que de vouloir et d'inventer. Tous les métiers plaisent autant que l'on y gouverne et déplaisent autant que l'on y obéit. Faire et non pas subir, tel est le fond de l'agréable. »

Sans prétendre faire une présentation exhaustive de l'histoire de ces ateliers et dépôts, quelques anecdotes peuvent permettre d'esquisser une réponse à cette question essentielle.

\section{Au dépôt de Mohon. 1955-1957}

Ce dépôt avait été choisi pour y affecter les machines conçues pour l'extension de la traction électrique en courant monophasé $50 \mathrm{~Hz}$ après sa première expérimentation en Savoie.

En effet ce dépôt était bien placé au milieu de la ligne industrielle Valenciennes-Thionville, était bien équipé (pont transbordeur de 50 tonnes) et n'était pas trop éloigné de Paris.

Mais les questions à résoudre étaient nombreuses et variées.

S'agissant d'une ligne à fort trafic industriel, il était hors de question de perturber la circulation des trains de manière sensible. Or, d'une part, la formation du personnel à la traction électrique s'adressait à des agents n'ayant que des connaissances essentiellement mécaniques, avec des particularités propres à la traction vapeur : conduite du feu, alimentation en eau... difficilement transposables en électricité ; d'autre part, la technologie des locomotives à courant alternatif à $50 \mathrm{~Hz}$ constituait une nouveauté avec, de surcroit, quatre séries de locomotives de conception différente ; leurs exigences d'entretien restaient à découvrir ; enfin, la constitution même des parties mécaniques était assez différente de celle de la majorité des locomotives électriques à courant continu en service à l'époque. Il s'agissait jusqu'alors de châssis dérivés des errements constructifs habituels de ceux des locomotives à vapeur sur lesquels on avait placé de l'appareillage et des moteurs électriques à la place des chaudières ; seul le mode de transmission aux roues différait sensiblement (quoique les bielles aient encore été utilisées sur quelques machines); les parcours entre révisions étaient d'ailleurs comparables.

Voilà le défi, et il n'était pas question d'échouer. Il est difficile, au moment du présent colloque, d'imaginer l'âpreté des discussions et des critiques soulevées à l'encontre de ce mode de traction ; bien que ces difficultés aient eu lieu essentiellement au niveau de la DETE (Division des études de traction électrique de la SNCF) dans ses rapports avec certains constructeurs ou réseaux étrangers, le personnel de Mohon en avait des échos et cet élément fut probablement un stimulant efficace. 
Les surprises pourtant ne manquaient pas :

- au retour d'un des premiers trajets, les capots de la première BB 12000 s'étaient retrouvés soudés à leurs armatures l'origine était simple... après qu'elle eut été décelée) ;

- les inverseurs des BB 13000 avaient une fâcheuse tendance à rester collés, et que dire de leurs multiples porte-balais et des balais hétérogènes ;

- les CC 14000, monotriphasées particulièrement complexes, ont présenté une multiplicité de pannes diverses, souvent mineures... mais pas toujours, aptes à décourager les plus astucieux dépanneurs (heureusement, ils n'avaient pas, comme Vatel, d'épée à leur disposition !) ;

- même les robustes CC 14100, comparables à des locomotives Diesel-électriques dont le moteur thermique aurait été remplacé par un moteur électrique synchrone, s'octroyaient quelques flashes sans parler des problèmes de condensation sur les groupes convertisseurs lors des périodes de redoux - en hiver, la neige poudreuse pénétrait par les orifices de ventilation, se condensait à l'intérieur et provoquait des amorçages. Les drogueries de la région ont été dévalisées en serpillères pour les opposer à cette invasion inopportune en les accrochant derrière les persiennes.

Les premiers temps furent donc consacrés à faire face aux difficultés à trouver une machine en état pour chaque train, et à connaître les machines au gré des surprises du jour. Inutile de préciser que les heures n'étaient pas comptées!

Mais, heureusement, une réflexion engagée à Bordeaux pour améliorer les conditions d'entretien des $141 \mathrm{R}$ avait débouché sur une nouvelle méthode d'organisation de l'entretien des locomotives, en parallèle avec un processus de formation des agents concernés, associant plus efficacement théorie et pratique (voir à ce sujet la communication de G. de Terssac et de K. Lalande). La participation active des agents d'entretien, ouvriers et maitrise, portait à la fois sur la détection des anomalies et des règles d'usure et sur la détermination des actions préventives à effectuer. De simples exécutants, ces agents devenaient acteurs. Ce sentiment pouvait particulièrement se développer dans cette phase active et vivante de découverte d'un nouveau matériel et de détermination concrète de ce qu'il était nécessaire de faire pour l'utiliser et l'entretenir à bon escient. L'évolution de l'organisation a ainsi coinncidé avec l'évolution technique du matériel.

Par ailleurs, la fréquence des passages d'ingénieurs ou techniciens de la DETE permettait généralement d'éclaircir rapidement des 
questions restées en suspens ou d'orienter des dispositions techniques dans un sens pratique pour l'entretien; il en était de même avec les représentants des constructeurs présents au titre de la garantie, mais aussi pour parfaire leur connaissance concrète du matériel livré dans son service quotidien.

Dans ces conditions, l'ambiance que j’ai connue à Mohon était tout à fait satisfaisante.

La pointe de variété et, souvent, la source d'amusement des agents, étaient fournies par les nombreux visiteurs, attirés par la nouveauté pour certains, soucieux de vérifier que les performances annoncées étaient bien réelles pour d'autres. Les méthodes variaient selon les nationalités : alors que nous cherchions à présenter un circuit logique de révision ou de réparation, certains s'égaillaient partout en désordre avec de multiples photographies, d'autres, soucieux que rien ne leur soit caché, progressaient par tranches successives du bâtiment sans tenir compte du circuit des pièces..., etc.

Dans l'ensemble, au cours de ces premières années, l'attrait de la nouveauté, l'intérêt de la participation à la connaissance de la vie du matériel et à la définition de ses règles d'entretien, agrémentés de la variété des visiteurs m'ont semblé concorder avec la réflexion liminaire du philosophe.

Cela n'excluait pas parfois quelques inquiétudes sur des effets méconnus des nouveaux engins moteurs, comme on l'observe généralement dans les situations innovantes. C'est ainsi qu'une question avait été posée très officiellement sur l'incidence du champ magnétique des transformateurs (placés sous la cabine de conduite des locomotives) sur la virilité des agents de conduite. Évidemment une telle question avait provoqué quelque surprise, et rencontré l'incrédulité ; or, si le champ magnétique était hors de cause, la réaction au maintien, dans les premiers mois, d'un régime alimentaire de type «traction à vapeur ", avec quelques tonnes de charbon à manipuler à la pelle, alors que les agents se retrouvaient assis dans une cabine, chauffée en hiver, et sans effort physique notable, pouvait avoir une incidence sur la tendance à l'assoupissement, donc sur la qualité incriminée. La question n'était pas sans fondement même si la formulation était inexacte!

\section{Au dépôt de Strasbourg. 1959-1960}

Le monophasé est « débroussaillé ». Les règles s’installent mais le service assuré en traction électrique évolue. Une pré-série de quatre 16500 est reçue à Strasbourg; ce sont des machines légères, peut-être 
trop : très rapidement sont observées des déformations, puis des ruptures de dents d'engrenage.

Le personnel préfère par tradition quelque chose de plus solide. Mais les modifications utiles sont apportées progressivement et le comportement général du matériel devient satisfaisant.

Pour les conducteurs, des problèmes de stabilité de caisse et les bruits des organes de suspension ne sont guère de leur goût, les surnoms donnés aux locomotives, tels que «les danseuses », apparaissent. Par contre, les exploitants apprécient leur souplesse d'emploi. Les discussions sont vives.

Puis les premières BB 16000 arrivent, à la satisfaction générale ; ce sont des grosses 12000 !

Mais l'une d'entre elles, quasi neuve, est gravement accidentée à Thionville : alors que la «doctrine » officielle de F. Nouvion est qu'il faut faire des machines simples, en quoi il a raison, il est plus que réticent pour faire des machines bicourant, en quoi il a tort. Or, le chef de division Traction de l'Est en est très demandeur pour la liaison MetzDijon, chargée surtout en trafic marchandises (fig. 1 et fig. 2).

Par ailleurs, la question de la jonction $1500 \mathrm{~V}$ continu / $25 \mathrm{kV}$ $50 \mathrm{~Hz}$ avait déjà donné lieu à des réflexions; quelques locomotives dites « petit bicourant » permettaient de circuler à puissance très réduite à Aix-les-Bains sous $1500 \mathrm{~V}$, mais elles ne répondaient nullement aux exigences de circulation à puissance analogue sous les deux types d'alimentation. À la fin de 1955, Louis Armand se déclarait opposé à la poursuite du $1500 \mathrm{~V}$ de Paris-Lyon jusqu'à Marseille ; mais le surcoût d'une machine bicourant étudiée à l'époque était important. Une électrification de la rive droite du Rhône jusqu'à Avignon et Nîmes en $1500 \mathrm{~V}$ et de la rive gauche en monophasé a été examinée mais de telles solutions étaient jugées trop chères et mal commodes ${ }^{2}$.

À la faveur de l'apparition de redresseurs au silicium adaptés aux exigences de traction, un petit groupe de «comploteurs » comprenant heureusement André Cossié dont l'imagination concrète et pratique est sans limite et un représentant de Schneider-Westinghouse, ici présent,

2- Voir A. Blanc, « Le journal professionnel de Marcel Garreau, 13 août 1940 - 6 octobre 1955. Du $1500 \mathrm{~V}$ continu au $25000 \mathrm{~V} \mathrm{50Hz}$, l'électrification du réseau ferrovaire français vue par l'un de ses principaux auteurs », in «Électricité et chemins de fer, cent ans de progrès ferroviaire en France par l'électricité », acte du 4e colloque de l'AHICF/ $10^{\mathrm{e}}$ colloque de l'AHEF (Paris, mai 1995), Revue d'histoire des chemins de fer hors série, 5 / Coll. « Histoire de l'électricité », x, Paris, 1997, p. 215-266, en particulier p. 256-258 (notes de sept.-oct. 1955). 

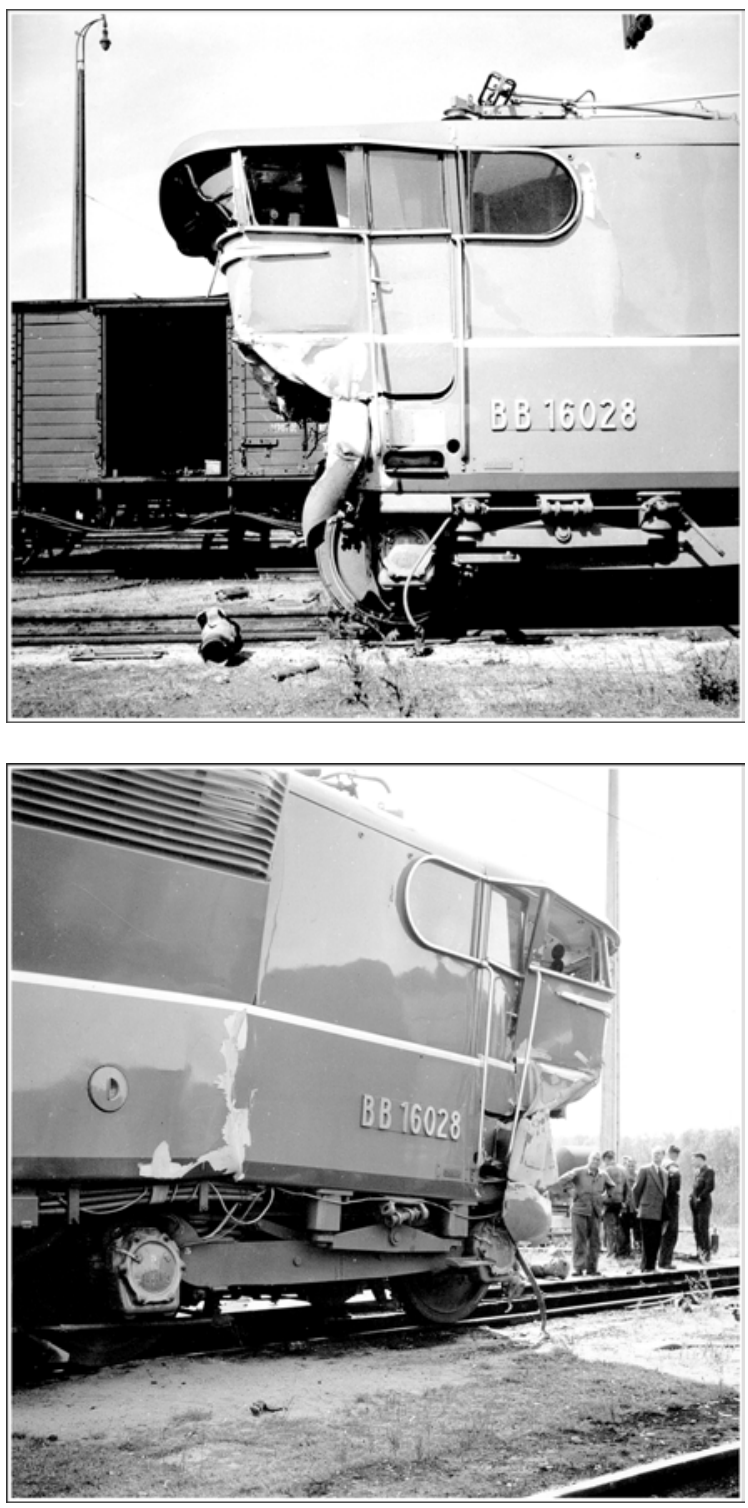

Figure 1 et 2 . La locomotive BB 16028, gravement accidentée en 1960, à Thionville, est réaménagée en prototype de locomotive bicourant $(25 \mathrm{kV}$ alternatif $1500 \mathrm{~V}$ continu) (le travail lui-même a été surtout fait à Hellemmes). Cl. et coll. A. Blanc. 
réalise la transformation de l'épave de la 16028 en une vraie bicourant (la 20005) avec la complicité active du dépôt de Strasbourg et des ateliers d'Hellemmes. Ce sera l'amorce des séries 25100 et 25200, séries bien réussies qui assureront une grande partie des services sous les deux tensions $25 \mathrm{kV}$ alternatif et $1500 \mathrm{~V}$ continu jusqu'à l'apparition des séries plus modernes de la catégorie $4400 \mathrm{~kW}$. Entre temps les 16500 elles-mêmes auront une descendance analogue avec les BB 17000, 8500, 25500 devant le succès de cette formule.

Dans un domaine infiniment plus modeste, le dépôt de Strasbourg devait envoyer chaque matin une locomotive haut le pied à Sélestat uniquement pour assurer le préchauffage de rames voyageurs en hiver. Un devis d'installation de prises fixes ayant paru exorbitant, le dépôt résolut de régler ce problème à sa façon avec les moyens locaux (fig. 3).

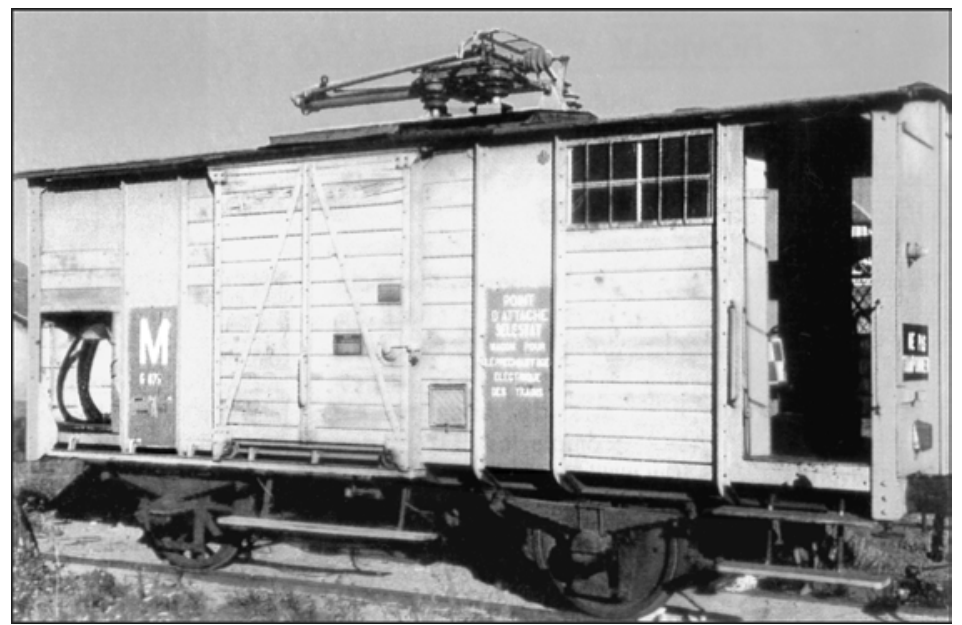

Figure 3. Le fourgon de chauffage des rames voyageurs "bricolé " par le dépôt de Strasbourg en 1960. Coll. A. Blanc.

Un pantographe AM 16 (prototype des modèles AM 18 qui sera largement utilisé par la suite), restait entreposé dans un coin du dépôt après les essais ; un transformateur de chauffage devenu sans emploi, un vieux wagon démodé promis à la ferraille généreusement donné par l'entretien de Hausbergen plus quelques bricolages pour réaliser l'appareillage de protection nécessaire... et voici un vieux wagon qui remplace avantageusement une locomotive pendant plus de 40 ans.

Il y a peu, l'auteur d'un article parlant de la réforme de ce wagon s'est demandé d'où il venait et pourquoi il était unique! 
Avec l'électrification de la ligne Paris-Strasbourg à partir de la province, le dépôt de Strasbourg héberge à son tour l'organisme d'études chargé de mettre en forme les documents d'entretien, précédemment situé à Mohon. L'application des méthodes d'organisation testées à Mohon s'effectue dans les mêmes conditions satisfaisantes avec les nouvelles séries de locomotives, elle constitue même une aide précieuse ; mais la variété est conservée avec de nombreux essais en vue de remédier aux problèmes d'instabilité des premières BB 16500, et avec l'expérimentation de prototypes bifréquences BB 20101 et 102 à moteurs directs $50 \mathrm{~Hz}$ ou 20103 - 20104, initialement dotées de redresseurs excitrons dont le fonctionnement était souvent aléatoire : ces redresseurs volumineux à refroidissement par air, d'un modèle prévu pour des installations fixes, se désamorçaient par déplacement du bain de mercure dans les courbes un peu prononcées. Les treize BB 20200, bifréquence, ne posèrent pas de problème particuliers.

La 20103 fut donc logiquement choisie pour expérimenter les premiers redresseurs secs au silicium. Ces machines terminèrent leur carrière en assurant des navette d'échange entre Strasbourg et Kehl.

Une autre particularité fut constituée par les essais de la première locomotive pour les chemins de fer indiens construite par Alsthom à Belfort. Comme ce réseau utilisait le frein à vide, les essais eurent lieu avec une BB 13000 assurant la fourniture d'air comprimé nécessaire au freinage du train d'essai (fig. 4).

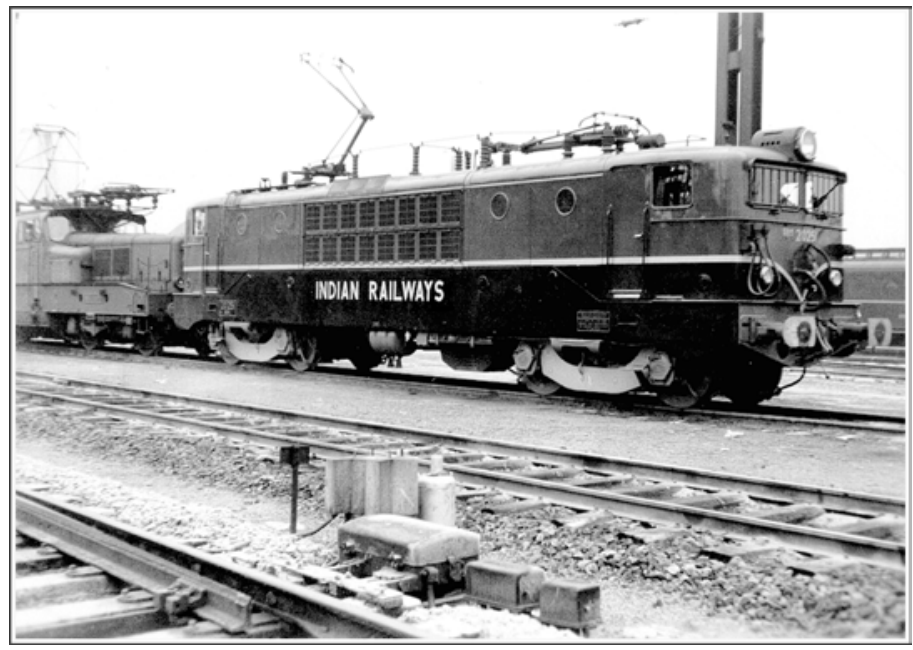

Figure 4. La première locomotive électrique fournie par Alsthom aux Chemins de fer indiens en essais au dépôt de Strasbourg, 1960. Cl. et coll. A. Blanc. 
Ainsi coexistaient le service courant, les mises au point et l'expérimentation des prototypes sur fond de rationalisation des méthodes qui a bien aidé le travail.

La question posée dans l'appel à communication à notre colloque trouve ici toute sa réponse : les dépôts sont à la fois un lieu de conservation des pratiques, de la connaissance des métiers et, en même temps, le champ d'application pratique de l'innovation technique et gestionnaire.

L'évocation de la vie au dépôt de Strasbourg en 1959-1960 serait incomplète si elle ne portait pas aussi sur son personnel.

À Strasbourg, comme ailleurs, la période de guerre n'alimentait guère les conversations, mais plus qu'ailleurs les souffrances endurées et les contraintes subies étaient profondément marquées dans les esprits. Quelques exemples : le chef du dépôt avait été interné au sinistre camp du Struthof pour avoir parlé français dans un tramway et n'avait dû son salut qu'à l'avance suffisamment rapide des troupes alliées qui avaient délivré les survivants ; un chef d'atelier avait été affecté à l'espèce de charrue (le "loup", Schienenwolf) qui brisait les traverses et coupait les rails pendant la retraite de la Wehrmacht sur le front de l'Est; un contremaitre racontait avec humour que, lors de la bataille du MontCassin, c'est sa petite taille qui l'avait protégé car ses adversaires visaient toujours trop haut ; un chef d'équipe, âgé seulement de 16 ans en 1945, avait cherché à éviter l'enrôlement forcé au cours des derniers mois des hostilités en se cassant volontairement le bras avec une barre de fer, ce qui ne l'avait pas empêché d'être envoyé sur le front de l'Est après un séjour en hôpital militaire ; il avait ensuite réussi à se faire capturer par les Américains en accompagnant les derniers jours de la retraite des unités allemandes en restant entre celles-ci et les troupes soviétiques !

Avec l'introduction des guides de dépannage sur les locomotives Diesel de manœuvre, une difficulté supplémentaire apparut pour les conducteurs de manœuvre les plus âgés : ceux nés aux alentours de 1910 avaient fait leur scolarité de base en allemand, habitaient souvent aux environs de Strasbourg où le dialecte alsacien était largement répandu et avaient de nouveau été obligés de parler uniquement allemand de 1940 à 1945. Inutile de préciser que la lecture du français avec suffisamment de précision dans les documents techniques a pu présenter bien des difficultés pour certains d'entre eux malgré leur bonne volonté ! 


\section{Au dépôt de Noisy-le-Sec. 1960-1962}

Au début des années 1960, trois dépôts alimentaient la région parisienne de l'Est : La Villette pour les voyageurs et la banlieue, Vaires pour le trafic marchandises et un peu de banlieue, et Noisy-le-Sec pour quelques express, la banlieue et surtout des trains de messageries. À l'approche de l'électrification, La Villette a été allégé de ses machines banlieue et des $241 \mathrm{P}$, ne conservant que les Pacific. Noisy a donc vu son parc de locomotives à vapeur fortement accru. À côté de la vapeur, il y avait le dépôt autorails ; selon un état de fait habituel à l'époque, ces deux unités opérationnelles distinctes coexistaient au sein d'un même établissement. Le dépôt «autorails » était multiforme : il comportait des autorails classiques pour services omnibus et quelques service directs, les RGP (rames à grand parcours) et TEE (Trans Europ Express), et les spéciaux : la presse et les autorails dits « de commandement». S’y ajoutaient quelques locomotives Diesel de manœuvre 030 DA (devenues C 61000) puis BB 63000/63500. La part thermique s'est étoffée progressivement avec l'arrivée des BB 66000 pour la banlieue, puis des locomotives Diesel de lignes 68000/68500 et les prototypes 69001/2 et 70000 .

Je n'omettrai pas les fourgons-chaudières de $800 \mathrm{~kg} / \mathrm{h}$ puis $1300 \mathrm{~kg} / \mathrm{h}$ qui accompagnaient les Diesel pour le chauffage des trains, ne fût-ce que par les soucis qu'ils nous causèrent !

Le dépôt de Noisy-le-Sec se trouvait donc à l'écart du grand mouvement de modernisation lié à l'extension de l'électrification, si ce n'est pour recevoir un surcroît de charge nécessairement momentané.

Du côté des locomotives Diesel de ligne, l'arrivée des premières unités - BB 66000 puis AA.AA 68000 et 68500 - ne se fit pas sans mal. Bien sûr, les servitudes de la vapeur s'estompaient, mais ces locomotives faisaient au mieux les performances des locomotives à vapeur qu'elles remplaçaient avec, les premiers temps, une qualité de service moindre en termes d'incidents. On était loin du prestige des locomotives électriques.

Ce n'était pas nécessairement très motivant pour le personnel!

Néanmoins, dans une situation qui n'était pas figée, ce personnel s'est adapté en permanence aux besoins évolutifs entre le surcroît de charge pour la vapeur encore prédominante mais dont le déclin s'amorçait, les reconversions à la traction thermique (et un peu à la traction électrique), les inévitables aléas des nouveaux Diesel et, cerise sur le gâteau, les déplacements présidentiels à l'époque de l'OAS. 
À côté de l'attrait de la nouveauté, tempéré par les ennuis techniques, j’ai ressenti chez beaucoup d'agents, qu'ils soient sédentaires ou roulants, le souci de finir la vapeur en beauté.

La relation par un mécanicien du service assuré avec une $141 \mathrm{P}$, écrite spontanément à cette époque, illustre de façon très vivante les conditions de travail, le lien avec le service d'entretien et la relation de tous les instants entre le mécanicien, le chauffeur et leur locomotive. Il n'y a pas trace de désintérêt vis-à-vis d'un mode de traction dont il savait parfaitement la fin proche (Annexe 1).

Sur l'Est, il n'y a pas eu de manifestation officielle de la fin de la vapeur sur Paris-Strasbourg, contrairement au Nord et à l'Ouest. La vapeur s'est simplement éteinte : le dernier express 12 en juin 1962 est arrivé, à l'heure bien sûr, à Paris-Est : devant l'équipe de la 241 P 26, quelques collègues, le chef de dépôt adjoint (j'étais à mon grand regret retenu par ailleurs), le chef de feuille, le chef d'atelier... et la page était tournée! Quelques années de survie sur la ligne de Mulhouse, la fin à Sarreguemines... Ce n'était plus le temps où le moindre événement déplaçait quelques dirigeants de haut niveau (fig. 5).

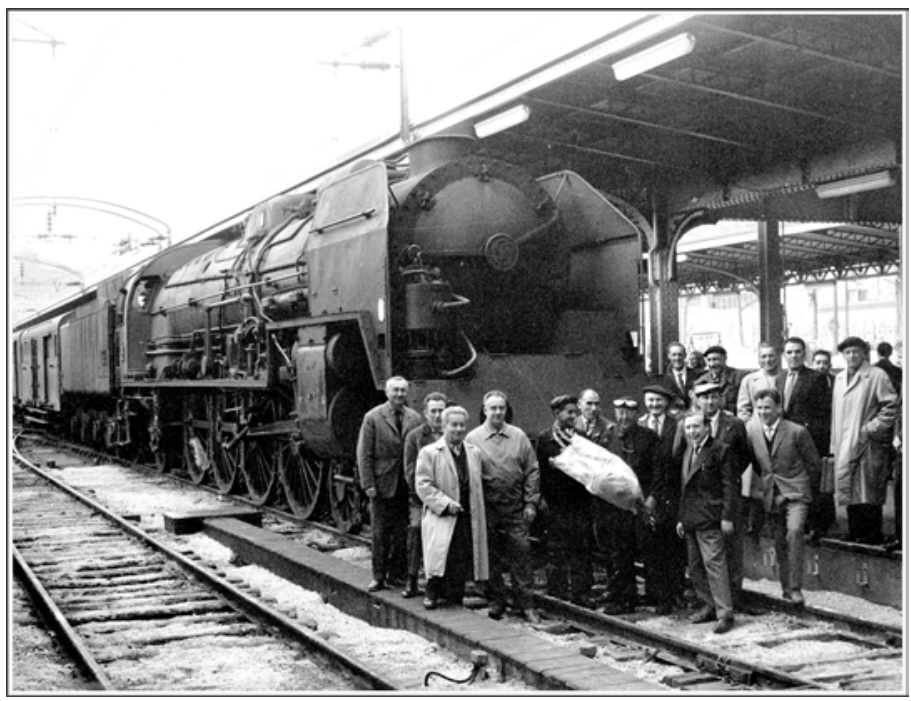

Figure 5. Arrivée en gare de I'Est du dernier Express StrasbourgParis tracté par une locomotive vapeur (241 P 26), juin 1962. L'équipe de conduite et l'encadrement du dépôt de Noisy-leSec posent devant la machine. Cl. P. Bernier. Coll. A. Blanc. 
Les anecdotes ont été nombreuses.

\section{Le service de la "Presse"}

Deux autorails De Dietrich avaient été transformés pour transporter dans la nuit les journaux parisiens à Nancy avec arrêts au retour à Châlons-sur-Marne et Épernay. L'un était en réserve et, en cas d'indisponibilité, une consigne prévoyait de fournir en toute priorité une $141 \mathrm{P}$ et des allèges postales. Une nuit, je suis réveillé dans mon premier sommeil par le chef de service de nuit ; le dialogue a été le suivant :

«- Chef! je ne peux pas sortir la Presse!

- Eh bien, sors la réserve!

- Oui, mais je ne peux pas sortir la réserve...

(Là, j'étais complètement réveillé)

- Trouves-en une à la rentrée.

- Oui, mais je ne peux pas en virer [dans le bon sens], il y a une machine dans la plaque!» (fig. 6).

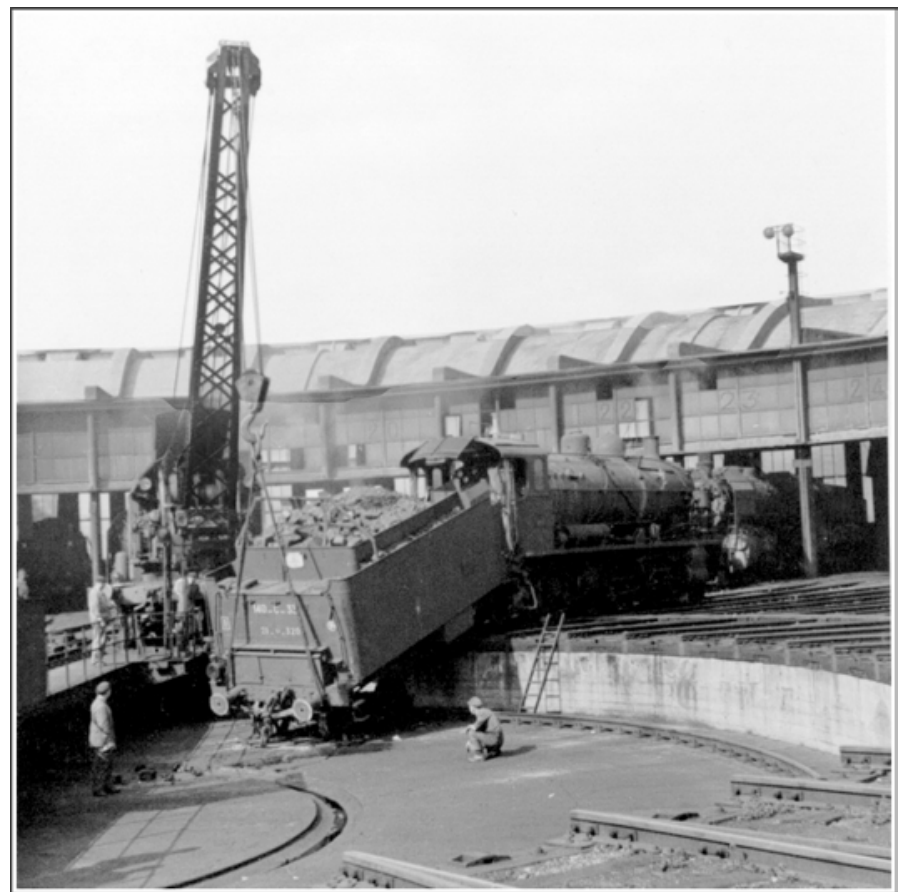

Figure 6. "Chef, il y a une machine dans la plaque !". Dépôt de Noisy-le-Sec, 1961. Coll. et cl. A. Blanc. 
C'était un peu la chanson «tout va très bien, Madame la Marquise ». Nous avons quand même réussi à sortir un autorail et la presse est partie avec peu de retard.

\section{Les fourgons-chaudières}

Les premiers modèles capables de vaporiser $800 \mathrm{~kg}$ à l'heure se sont rapidement révélés un peu justes en capacité, d'autant qu'une mầtrise encore approximative du traitement de l'eau nécessaire fut à l'origine de nombreux cas d'entartrement des chaudières ; en outre, leur groupe électrogène était lancé au moyen d'un démarreur à inertie qui nécessitait de nombreux tours de manivelle à vitesse progressivement croissante. Par temps froid, cet exercice devenait assez difficile ; en cas de raté de démarrage, deux ou trois essais successifs devenaient épuisants. Dans ces conditions le chauffage des premiers trains de banlieue du matin devenait d'autant plus aléatoire que la température était plus basse! Faut-il préciser que ce matériel n'était guère apprécié de ses utilisateurs et des clients. Heureusement des fourgons de plus grande capacité $1300 \mathrm{~kg}$ - à démarrage électrique sont venus prendre la relève, puis le chauffage électrique s’est progressivement généralisé.

\section{Les autorails dits "de commandement"}

Ces autorails dotés d'aménagements particuliers avec salons (à vrai dire de simples bureaux), chambres et une petite cuisine n'assuraient aucun service commercial et étaient conduits par quelques conducteurs de bon niveau dont l'un était toujours disponible pour permettre un départ dans des délais très brefs. Deux d'entre eux, des De Dietrich, étaient particulièrement affectés aux déplacements de personnalités, surtout le 2511 dit Présidentiel (fig. 7), l'autre le 2514 assurant plutôt des déplacements (rares) du directeur général ou des directeurs de réseaux (les 2513 et 2515, de construction analogue, avaient été affectés au service de la presse, le 2512 ayant disparu pendant la guerre) (fig. 8).

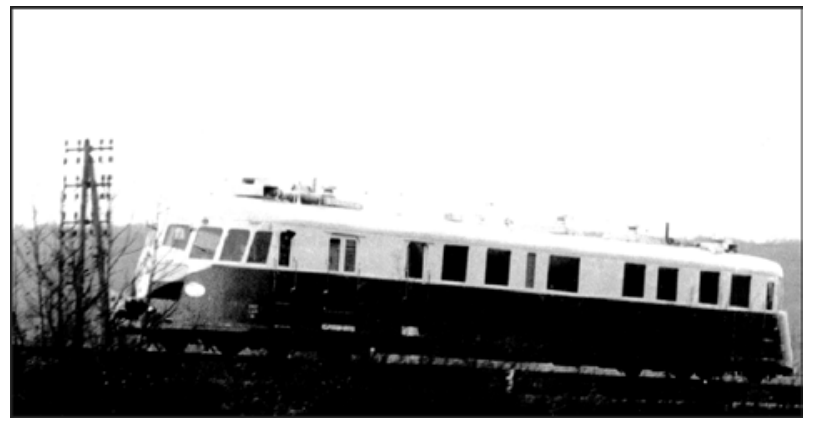

Figure 7. L'autorail présidentiel De Dietrich 2511. Cl. SNCF. Coll. A. Blanc. 


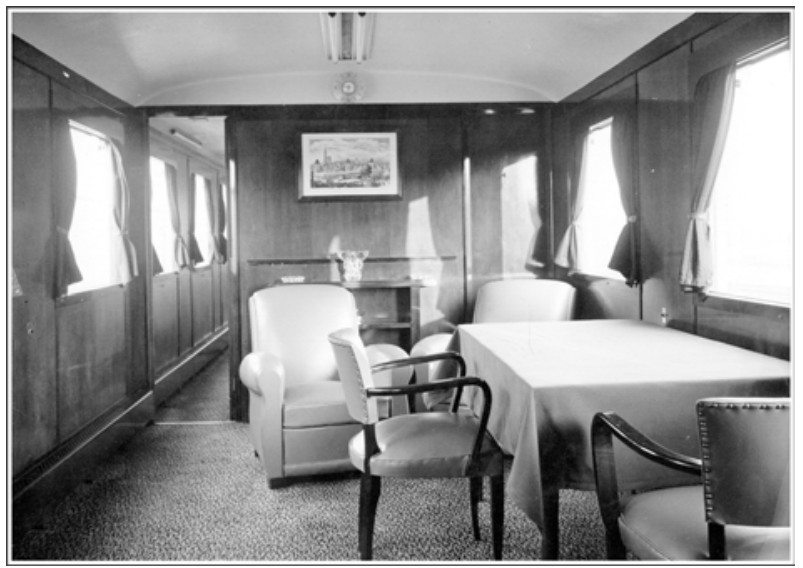

Figure 8. L'aménagement intérieur de l'autorail présidentiel De Dietrich 2511 réalisé par les ateliers de Noisy-le-Sec (sièges et table sont surélevés...). Cet autorail a été confié en 1998 par le Musée français du chemin de fer à I'Association De Dietrich pour restauration. $\mathrm{Cl}$. et coll. A. Blanc.

Un autorail Renault AEK 7011 (fig. 9) était utilisé principalement pour des tournées de surveillance de l'état de la voie ; il a dû circuler sur l'essentiel des ligne de la SNCF. À sa réforme, il a été remplacé par deux autorails " Picasso » X3800, aménagés dans des conditions analogues.

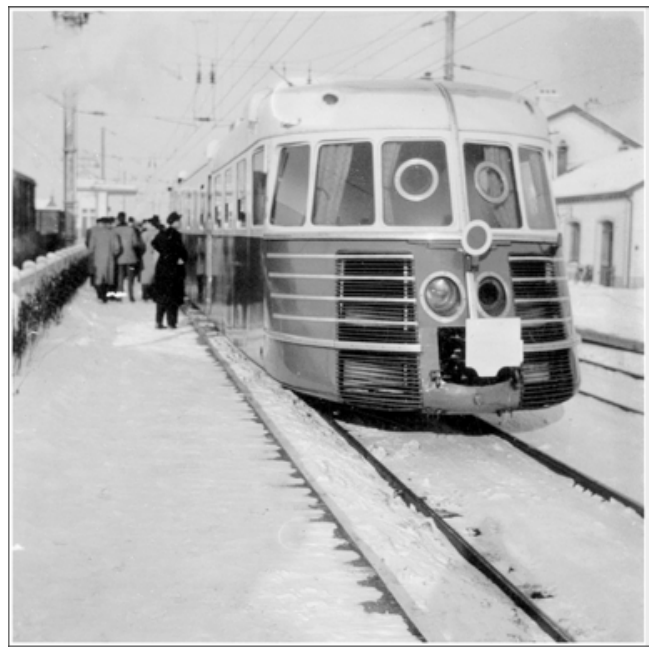

Figure 9.

L'autorail de service 7011 (Renault AEK) en gare d'Avesnes. Voyage de présentation des installations $50 \mathrm{~Hz}$ (décembre 1954). Cl. SNCF. Coll. A. Blanc. 
Étant donné la nature des services assurés, le personnel intéressé devait posséder, outre bien entendu une très bonne compétence technique, des qualités de discrétion et d'initiative propres à faire face à des situations variées autant qu'imprévues. Le président Coty avait souvent utilisé le 2511 pour se rendre en Normandie ; le général de Gaulle l'emprunta plusieurs fois pour rejoindre Colombey-les-Deux-Églises, surtout pendant la période des attentats de l'OAS durant laquelle il variait ses moyens de transport de façon inopinée. Pour les voyages officiels, la SNCF mettait à sa disposition des rames à grand parcours RGP ou TEE ou, bien sûr, la voiture présidentielle. Si la circulation de ces trains, bien programmée et surveillée, n’a pas donné lieu à beaucoup d'incidents (hormis le mémorable voyage du président Auriol dans les Vosges !), les petits « à-côtés » étaient plus imprévisibles.

Ainsi, lors d'un voyage à Fécamp du ministre des Transports, M. Robert Buron, à l'occasion du grand pardon des Terre-Neuvas, le conducteur (M. Malozzi) constate une importante fuite d'eau à un radiateur du 2511. Arrivé à Fécamp, il réussit, avec l'aide d'agents de la gare, à démonter le radiateur, pourtant bien volumineux et lourd, dans une fosse sous l'autorail, l'emporta sur une brouette chez un garagiste pour faire reboucher le trou et le remonta à temps pour accueillir le ministre et assurer son retour dans de bonnes conditions. À la rentrée au dépôt, il me précisa qu'il avait bien eu le temps de se laver les mains !

À l'occasion d'une « circulation balai » devant la RGP présidentielle, le conducteur du 2511 perçut un claquement sec qu'il pensa dû à une explosion sur la voie ; freinant aussitôt, il s'aperçut que l'efficacité du freinage de l'autorail était fortement diminuée ; l'origine possible du bruit perçu étant changée, il continue à vitesse modérée jusqu'à la prochaine gare où il demande en hâte à l'agent, présent sur le quai, de le garer rapidement sur une voie de service; ce qui fut fait et l'autorail présidentiel passa en ayant seulement amorcé un freinage à la vue du signal d'entrée en gare fermé qui put être réouvert à temps pour éviter un arrêt. Il s'agissait d'une rupture d'une chandelle de suspension qui avait entrainé l'arrachement du tuyau d'alimentation d'un cylindre de frein.

La restauration à bord fût une autre source de surprises : on avait commencé dès 1946 à servir au général de Gaulle un sauté de veau Maréchal, ce qui était inopportun à l'époque ; mais le cuistot s'appelait Maréchal et avait une recette qui lui était propre ; il n'avait pas vu malice à le porter sur le menu. 
Quelques jours après l'attentat de Petit-Clamart, le même général de Gaulle trouva sur son menu de dîner, au départ de Paris, un potage de petits pois à la Clamart... du meilleur goût! Le menu avait été approuvé à l'avance par les services du protocole, était bien entendu resté confidentiel et personne n'a relevé l'anomalie au dernier moment, sauf évidemment le principal intéressé. Le maitre d'hôtel de la rame TEE en était encore livide à son retour.

Dans la même période, je reçois un samedi matin une communication directe du chef d'arrondissement m'informant d'un départ du Président avec le 2511 ; l'autorail est préparé et au moment de son départ pour la gare de l'Est, un second coup de téléphone me précise le point d'arrêt prévu : le passage à niveau de Longchamp sur la $\mathrm{RD} 15$ avec indication du point kilométrique sur la voie. Je répercute ces informations au conducteur qui part aussitôt gare de l'Est prendre son voyageur et les quelques personnes qui l'accompagnaient, et repart selon un processus discret et parfaitement chronométré. Après m'être assuré que le voyage s'était bien passé, je rejoins mon domicile... pour être rapidement rappelé au dépôt par le commissariat spécial de l'Élysée ; il m'apprend que des indiscrétions avaient eu lieu sur le choix du point d'arrêt et me demande si je pouvais fournir des explications. Evidemment, je ne le pouvais pas.

Quelques temps après, j’ai appris que la SNCF n'y était pour rien, mais que la gendarmerie, étant venue à l'avance reconnaitre les lieux, avait involontairement attiré la curiosité de la garde-barrière et pour expliquer sa présence inhabituelle sur ce chemin bien tranquille avait laissé entendre que... Moyennant quoi, le Président avait été reçu avec tous les égards dus à sa fonction par les notabilités du secteur, les enfants des écoles... etc.

Lors d'un déplacement du général dans la région d'Amiens, un agent de la gare du Nord surprend une conversation à voix basse entre deux personnes inconnues de lui, peu après le départ du 2511 : «Estce que la bombe a été bien chargée ? " Un peu inquiet, il hésite, puis décide d'en informer ses chefs ; l'autorail avait alors effectué près de la moitié de son parcours. Affolement général, on réussit à joindre par radio les responsables de la sécurité à bord de l'autorail ; ils procèdent discrètement à un examen, sans résultat. Compte tenu de l'avancement du voyage, la décision est prise de le terminer normalement. À titre d'information, le dessert chargé à bord était... une bombe glacée.

L’autorail 2511 avait aussi été utilisé par le général de Lattre de Tassigny pendant l'hiver 1944-1945 durant les derniers mois des hostilités. Étant rentré passablement trempé par la pluie, son ordonnance 
cherchait comment faire rapidement sécher ses chaussures. Pour ce faire il plaça celles-ci sur la porte du four de la cuisine en la laissant ouverte... sauf que les secousses en marche la refermèrent! Au bout de peu de temps le crêpe des semelles avait quelque peu fondu et l'ordonnance craignait les foudres de son chef ! Malozzi sortit alors son couteau, découpa le crêpe et remit les semelles au gabarit du reste de chaque chaussure. Il complète même son travail en découpant des étoiles sous la chaussure avec la pointe de son couteau ; il remit son travail à l'ordonnance en précisant que, de cette façon, on en connaitrait sans hésitation le propriétaire!

En même temps que les locomotives Diesel de ligne 6800068500 étaient mises en service, deux couples de prototypes furent affectés à Noisy.

Les BB 69001 et 2 à transmission hydraulique, remarquables engins de $90 \mathrm{t}$ pour plus de $4000 \mathrm{cv}$. Ils effectuèrent un excellent service avec une grande fiabilité ; malheureusement leur inaptitude au chauffage électrique les confina à de rares exceptions près dans un service, obscur mais bien utile, de traction de trains de marchandises lourds, surtout les trains complets d'eau de Vittel, entre les Vosges et la région dijonnaise.

Les CC 70001 et 2, plus compliquées, à transmission électrique utilisant un alternateur birotor (les deux parties tournaient en sens inverse l'une de l'autre) ne sortirent par contre guère de leur rôle de prototype expérimental et disparurent dès la mise en service des CC 72000.

Noisy-le-Sec avait donc aussi une part dans l'expérimentation de prototypes en même temps qu'il avait en charge de mettre en service les nouvelles locomotives Diesel de série, dans des conditions d'organisation de leur entretien largement inspirées de celles choisies pour les locomotives électriques monophasées.

Le regroupement des deux structures d'ateliers d'entretien, vapeur et thermique, fut alors effectué, des locaux fonctionnels furent aménagés, essentiellement avec les moyens de l'établissement, dans une partie de la rotonde devenue surabondante ; tout était prévu pour accueillir convenablement les nouvelles locomotives Diesel en plus grand nombre ; le personnel a joué le jeu. On avait même commencé à embellir le dépôt en semant du gazon dans de la terre de lavage de betteraves déversée dans la cuve de l'ancien pont tournant de la rotonde détruite par les bombardements de juillet 1944. 
C'est alors que la décision fut prise de faire de Chalindrey le gros dépôt Diesel qui était nécessaire pour couvrir les besoins du secteur non électrifié, et non pas Noisy. Plus que technique, cette décision répondait à des considérations humaines : le personnel de Noisy pouvait aisément être employé aux ateliers du Matériel voisins ou au dépôt de La Villette alors qu'à Chalindrey et chez son proche voisin, Chaumont, c'était le vide en matière de possibilités d'emploi.

Le dépôt de Noisy s'est donc progressivement effacé ainsi, par la suite, que les ateliers voisins, un chantier multitechniques a vu le jour sur une partie de ses emprises, mais c'est maintenant un centre d'entretien des rames automotrices de banlieue qui a redonné une nouvelle vie à ces installations ferroviaires.

Comme conclusion, il convient de souligner le rôle essentiel de l'encadrement et de la maitrise de terrain au cours de cette période de mutation. Non seulement ils se sont largement adaptés aux nouvelles techniques mais dans leur grande majorité ils ont accompagné, voire impulsé, les changements d'organisation, donc souvent de rôles, qui étaient préconisés.

Une mention particulière est due aux chefs de Traction et aux inspecteurs de Traction dont j’ai pu apprécier, à cette époque et aussi plus tard, le dévouement, la compétence et la loyauté. 


\section{Annexe 1}

\section{Daniel Lécheneau : L'utilisation des $141 \mathrm{P}$ affectées au dépôt de Noisy-le-Sec}

Daniel Lécheneau a été, entre autres, mécanicien au dépôt de Noisy-le-Sec, affecté aux 141 P à l'époque de la fin de la vapeur. Chef de traction à Vaires en 1967 il a terminé sa carrière comme chef de traction principal (CTRAP) au dépôt de La Villette en 1973. Ce texte a été rédigé vers 2000 à partir des notes prises par l'auteur à l'époque des faits qu'il relate.

De 1946 à 1950, le dépôt de Noisy-le-Sec reçoit les 141 P dont beaucoup sortent d'usine. L'effectif atteint environ 38 machines, toutes titulaires, c'est-à-dire affectées chacune à une équipe : mécanicien plus chauffeur, et réparties en deux roulements. Le roulement $n^{\circ} 1$, réservé à l'élite du dépôt, est baptisé " grand roulement » ou « roulement des seigneurs » ou " roulement des chouettes », vu le nombre de nuits effectuées (11 périodes de nuit sur 14 de travail). Le parcours moyen journalier est de $308 \mathrm{~km}$, soit $4312 \mathrm{~km}$ pour les 14 périodes de travail. C'est le roulement noble du dépôt mais c'est un roulement très difficile.

L'ensemble des trains remorqués sont des "messageries ", directs et lourds, dont la charge varie de 700 à 1000 tonnes.

Ses parcours : de Noisy à Charleville, Noisy à Metz, Noisy à Blainville puis Sarrebourg, Noisy à Chalindrey, Chalons à Reims, Juvisy à Bar-le-Duc. Un aller et retour « trains express » est assuré sur Paris, Chalindrey (trains 409 et 410).

Le roulement $\mathrm{n}^{\circ} 2$ compte également 14 périodes de travail, assure des messageries et des marchandises directs.

Ses parcours : de Noisy à Reims, Noisy à Chalons et Bar-leDuc, Noisy à Troyes, Reims, Chalons, Juvisy à Bar-le-Duc.

Néanmoins, les machines affectées à ce roulement peuvent assurer le roulement $\mathrm{n}^{\circ} 1$ en cas de besoins ou la remorque de trains rapides ou express supplémentaires aux fêtes et vacances, notamment en fin d'année, trains de skieurs à destination du réseau Sud-Est (détournés par Chalindrey) de la Suisse et de l'Autriche. Il est à noter qu'en ces périodes de fin d'année des $141 \mathrm{P}$ des dépôts d'Argentan et du Mans étaient prêtées au dépôt de Noisy pour assurer ce trafic. 


\section{Trains de messageries directs $n^{\circ} 4017$ et $n^{\circ} 4008$. Étape Noisy-le- Sec à Sarrebourg et retour}

Les trains de messageries directs 4017 et 4008 remorqués par les $141 \mathrm{P}$ de Noisy (roulement $\mathrm{n}^{\circ} 1$, journée 11 et 12), parcours Noisytriage à Sarrebourg, donnent une consommation approximative en eau et charbon de ces locomotives.

La distance de Noisy-triage à Sarrebourg est de $415 \mathrm{~km}$ par le contournement de Nancy, ligne 27 de Toul à Blainville via Chaligny, et Neuves-Maisons, itinéraire emprunté à l'époque par l'ensemble des trains de messageries et de marchandises car plus court de $8 \mathrm{~km}$ que via Nancy, mais rendu plus difficile par le profil et par trois limitations de vitesse permanentes situées au pied des rampes, $30 \mathrm{~km} / \mathrm{h}$ à Toul ligne 1 (ligne 27), $40 \mathrm{~km} / \mathrm{h}$ à Chaligny (bifur ligne 27 / ligne 14 Nancy-Mirecourt), $30 \mathrm{~km} / \mathrm{h}$ à Blainville (ligne 27 / ligne 1).

\section{Le 4017}

Prise de service 21 heures $^{3}$ à Noisy triage, départ 21 h 40, Sarrebourg arrivée $3 \mathrm{~h} 55$, fin de service $4 \mathrm{~h} 35$. Train complet à destination de Strasbourg-Cronenbourg sans modification de composition en cours de route. Référence : 700 tonnes, 770 tonnes admises $(10 \%$ de surcharge). Marche tracée à $90 \mathrm{~km} / \mathrm{h}$ de Noisy à Vitry-le-François puis à $80 \mathrm{~km} / \mathrm{h}$ de Vitry à Bar-le-Duc. De Bar à Sarrebourg, marche tracée à 70 - $80-90 \mathrm{~km} / \mathrm{h}$ selon le profil. Ce train a souvent une composition de 35 à 40 wagons pour une charge comprise entre 700 tonnes et 770 tonnes. Un arrêt à Bar-le-Duc pour prise d'eau (6 minutes).

\section{L'étape de Noisy - Bar-le-Duc}

Les $245 \mathrm{~km}$ étaient effectués sans prise d'eau ce qui impose de sortir du dépôt avec un tender bien complété en eau $\left(34 \mathrm{~m}^{3}\right)$ et une bonne utilisation de la machine en cours de route, c'est-à-dire chaudière maintenue au timbre, sans levée de soupapes, une marche d'admission relevée si possible à $20 \%$, un régulateur ouvert en grand au cours de cette étape. À l'aide des robinets de jauge tender, je vérifiais au Pk 120, peu après Dormans, que l'eau coule même faiblement au robinet des $20 \mathrm{~m}^{3}$, de même, au Pk 170 avant Chalons, au robinet des $15 \mathrm{~m}^{3}$, au Pk 236, avant Revigny, au robinet des $10 \mathrm{~m}^{3}$ (dernière gare où il était possible de faire un complément d'eau) et Bar-le-Duc est atteint sans problème. À l'arrêt à Bar-le-Duc il restait 7 à $8 \mathrm{~m}^{3}$ d'eau, ce qui donne une consommation de $26 \mathrm{~m}^{3}$, soit 105 à 110 litres au kilomètre parcouru.

3- Machine équipée par les soins du dépôt. 


\section{Étape Bar-le-Duc à Sarrebourg (170 km)}

Compte tenu du profil et de trois limitations permanentes de vitesse (30 - $40-30 \mathrm{~km} / \mathrm{h})$, la consommation était de 22 à $23 \mathrm{~m}^{3}$, soit 135 litres au kilomètre environ.

\section{La consommation de charbon}

Une consigne du dépôt de Noisy invitait les mécaniciens assurant la remorque du train 4017 de Noisy-Sarrebourg à compléter le tender en charbon avant la sortie du dépôt ${ }^{4}$. Ce que j'ai toujours fait, me permettant ainsi de joindre Sarrebourg sans soucis de manque de houille. Si le parcours de Noisy à Bar-le-Duc ne présentait guère de difficultés au point de vue conduite, par contre celui de Bar à Sarrebourg demandait de la part du mécanicien une bonne connaissance de la ligne et de son profil pour réaliser une marche économique.

À Sarrebourg, la charge était comprise entre $8500 \mathrm{~kg}$ et $9000 \mathrm{~kg}$, soit une consommation de $21 \mathrm{~kg}$ au kilomètre.

\section{Le train 4008}

Prise de service à 21 h 16, Sarrebourg départ 22 h 16, Noisy triage, arrivée $4 \mathrm{~h} 54$, fin de service 5 h 15 . Le train est complet de Strasbourg-Cronenbourg à Noisy-triage. Une dizaine de wagons de Noisy à Paris-Pajol. Sans modifications de composition de Strasbourg à Noisy. Référence : charge de 900 tonnes, 990 tonnes admises (10\% de surcharge). Marche moins tendue que le 4017 (19 minutes de plus) tracée à $85 \mathrm{~km} / \mathrm{h}$, un arrêt à Bar-le-Duc pour prise d'eau. Ce train avait toujours une composition de 50 à 54 véhicules pour 108 essieux, une charge comprise entre 980 et 990 tonnes et quelquefois plus (supplément non compris au bulletin de composition, des tonnes tractées à l'œil par la traction) $)^{6}$. Ce train malgré sa charge avait une bonne réputation de bien rouler, il est vrai que le profil était favorable.

La consommation en eau de Sarrebourg à Bar-le-Duc approchait de $20 \mathrm{~m}^{3}, 118$ litres au kilomètre, de Bar-le-Duc à Paris-Pajol plus HLP Noisy dépôt soit $261 \mathrm{~km}$, elle était de $22 \mathrm{~m}^{3}, 84$ litres au km, la charge en combustible au dépôt de Noisy variait de 7000 à $7500 \mathrm{~kg}$, soit $17 \mathrm{~kg}$ au kilomètre, compte tenu des $750 \mathrm{~kg}$ environ utilisés pour l'allumage et la mise sous pression à Sarrebourg. La consommation pour la remorque du 4008 était de $16 \mathrm{~kg}$ au kilomètre.

4- De 1000 à $1200 \mathrm{~kg}$ environ.

5- Équipe relevée à l'arrivée au triage.

6- La remise des écritures du train permettait aux mécaniciens de contrôler la charge.

Dans le cas présent, elle atteignait quelquefois 1100 tonnes. 
Les consommations indiquées, bien qu'approximatives, concernent l'ensemble des $141 \mathrm{P}$ de Noisy que j'ai conduites pendant cinq ans. Jeune mécanicien, je n'étais pas titulaire d'une machine, mais j'assurais de nombreux remplacements de mécaniciens titulaires du roulement $n^{\circ} 1$ utilisés comme faisant fonction CTRA ou en congé, ce qui m'a permis d'apprécier la valeur de cette noble série de locomotives.

Les parcours Noisy-Sarrebourg avec les charges précitées étaient certainement les plus longs de la SNCF de trains de messageries remorqués par des locomotives chauffées au charbon sans complément de combustible en cours de route. En comparaison une $141 \mathrm{R}$, bien qu'excellente locomotive, remorquant le 4017 ou le 4008 sur la même étape, aurait effectuée deux ou trois arrêts supplémentaires pour prise d'eau, et rentrée au dépôt de Chalons ou Bar-le-Duc pour faire le plein de charbon, malgré une capacité de son tender égale en combustible au tender 34P.

Cette série de 318 unités est la plus importante des locomotives à vapeur de la SNCF conçues par ses ingénieurs et construites entièrement par l'industrie française. Malheureusement, elles furent toutes ferraillées, seul un bogie bissel ZARA figure au musée de Mulhouse.

\section{Profil de la ligne de Noisy à Sarrebourg}

Noisy-le-Sec, Pk 9, altitude 56 mètres, à Bar-le-Duc altitude 184 mètres, distance $245 \mathrm{~km}$.

1- De Noisy $\mathrm{Pk} 9$ au $\mathrm{Pk}$ 55, succession de paliers, pentes, rampes de 3, 4, 5 pour mille ; la ligne rejoint la vallée de la Marne à Chelles.

2- Du Pk 55 à Vitry-le-François Pk 205, distance 145 km, paliers et rampes de 1 pour mille, pas de pente, la ligne remonte la vallée de la Marne.

3- De Vitry-le-François à Bar-le-Duc, Pk 254, distance $49 \mathrm{~km}$, $36 \mathrm{~km}$ en rampe de 2 à 5 pour mille, $4 \mathrm{~km}$ en pente de 3 à 5 pour mille, la ligne suit la vallée de la Saulx de Vitry à Sermaize puis celle de l'Ornain, de Sermaize à Nançois Tronville.

\section{Parcours de Bar-le-Duc à Sarrebourg}

1 - De Bar-le-Duc à Toul distance $66 \mathrm{~km}, 29 \mathrm{~km}$ en rampe de 3, 4, 6 et 8 pour mille dont $19 \mathrm{~km}$ en pente de 3, 4, et 6 pour mille, $10 \mathrm{~km}$ en rampe de 8 pour mille de Nançois-Tronville à Ernecourt - Loxeville et $11 \mathrm{~km}$ de pente de 8 pour mille d'Ernecourt-Loxeville à Lérouville. La ligne Paris-Strasbourg atteint son point culminant à Ernecourt-Loxeville, altitude 304 mètres. La ligne suit l'Ornain de Barle-Duc à Nançois-Tronville, franchit le seuil de partage des eaux se 
déversant dans la Manche et la Mer du Nord à Ernecourt. Elle suit la vallée de la Meuse de Lérouville à Pagny-sur-Meuse puis atteint la vallée de la Moselle peu avant Toul.

2 - De Toul à Blainville, ligne 27 via Chaligny-Neuves-Maisons, distance $48 \mathrm{~km}, 24 \mathrm{~km}$ en rampe continue de 2, 3, 4 pour mille et 5 pour mille, $15 \mathrm{~km}$ en pente de 2, 4, 5 pour mille, franchit la colline qui sépare les vallées de la Moselle et de la Meurthe qu'elle atteint à Rosières aux Salines.

3 - Blainville, altitude environ 184 mètres, à Sarrebourg Pk 431,8, altitude 250 mètres, distance $56 \mathrm{~km}, 26 \mathrm{~km}$ en rampe de 2, 3, 4 pour mille dont $19 \mathrm{~km}$ en rampe continue de 4 pour mille. La ligne suit le cours de la Meurthe de Blainville à Lunéville, puis atteint la gare de Igney-Avricourt (ancienne gare frontière allemande) à l'altitude de 295 mètres au $\mathrm{Pk} 415$ peu après Réchicourt-le-Château, puis descend sur Sarrebourg et rejoint la vallée de la Sarre à Héming.

\section{Le 409 - 1957 à 1960}

\section{Train express ligne 4 Région de Paris-Est de Paris à Culmont-Chalindrey}

Référence de marche : 241 A, 500 tonnes de Paris à Troyes; $231 \mathrm{C}, 400$ tonnes de Troyes à Culmont. Ce train a une marche très tendue de Paris à Troyes. La remorque est assurée par une $141 \mathrm{P}$ titulaire en équipe du dépôt de Noisy-le-Sec, journée 6 du roulement $n^{\circ} 1$. C'est le train drapeau du dépôt. Rentré le matin de Reims avec le train 4026 (fin de service $2 \mathrm{~h} 20$ ), j'assure la suite du roulement avec la $141 \mathrm{P}$ 250 et le chauffeur Jean Forest. Prise de service 16 h 25, haut le pied, départ de Noisy à 17 h 20, arrivée à Paris-Est à 17 h 42 pour remorque du train 409, départ 18 h 22.

$15 \mathrm{~h} 30$ passage à la commande, Jean est arrivé, nous consultons la feuille de roulement et le tableau SM (signalisation modifiée). Nous assurons notre présence au chef de feuille que nous saluons. Il nous confirme que nous "serons repos » au retour et suite du roulement (4045 pour Chalindrey).

Nous nous rendons au gril de sortie, La P 250 est sur fosse noyée. L’allumeur vidange les cendriers. Quel travail pénible que font ces allumeurs, un vrai métier de bagnard. Nous plaçons nos affaires personnelles dans les coffres arrières du tender (côté gauche pour le mécanicien, côté droit pour le chauffeur). Dans la cabine, coup d’œil aux manomètres, purge des niveaux d'eau, contrôle de la hauteur d'eau, dans le foyer contrôle des plombs fusibles, ils doivent être exempts de 
fuite. Vérification de l'état de propreté de la grille du foyer, puis de l'approvisionnement en eau du tender. La P 250 est conduite sur fosse de préparation, avec mise en position des bielles pour le graissage. Les bielles côté gauche sont mises en position pour le graissage : à $45^{\circ}$ côté avant de la machine, dans cette position les bouchons de graissage des bielles sont tous accessibles et notamment ceux des grosses têtes de bielles basse pression. Il faut faire le plein d'huile à la lampisterie : $30 \mathrm{~kg}$, dont $10 \mathrm{~kg}$ huile mouvement, $10 \mathrm{~kg}$ d'huile haute surchauffe VX $4,5 \mathrm{~kg}$ d'huile mouvement M3 pour les bielles, $5 \mathrm{~kg}$ d'huile de récupération, sans oublier de changer les « épongeurs » ou « torchettes » et de prendre des déchets de laine pour astiquage du corps cylindrique : un vrai chargement.

\section{Le graissage}

Ce travail est partagé, c'est une règle à la région Est. Le mouvement extérieur est graissé par le mécanicien, le mouvement intérieur et les dessous de boîtes de roues graissés par le chauffeur. Personnellement, je participe au graissage des dessous de boites (le chauffeur n'est pas un larbin). Point particulier de cette série de locomotives : les grosses têtes bielles basse pression sujettes au chauffage sont pré-graissées à l'aide d'une seringue. Le temps passe vite, l'heure de sortie approche. Nettoyage de la cabine, des bidons d'huile, burettes... Contrôle du fonctionnement des freins, des appareils d'alimentation de la chaudière, puis c'est l'appel classique de l'aiguilleur du poste 0 de sortie : « P 250 HLP Paris pour 409 en route. » Au cours du parcours HLP, Jean garnit les flancs et coins arrières du foyer en charbon, seule méthode pour maintenir un feu convenable pendant le stationnement de 30 à 40 minutes en gare de l'Est pour éviter des émissions de fumées, vu le combustible utilisé. C'est un problème pour les locomotives équipées d'un chargeur mécanique ou «stoker». Peu avant la gare de l'Est, un grand panneau «Évitez la fumée et le bruit » rappelle ces obligations aux mécaniciens et chauffeurs. De plus, aux heures de pointe, un inspecteur de traction ou chef-mécanicien (baptisé "caporal fumées ») est chargé de faire respecter ces mesures. Selon sa sévérité, cela se traduit par des observations, ou une sanction (7P1 + DSP pour les initiés).

$17 \mathrm{~h} 42$ arrivée en gare de l'Est sur voie 8. Le parcours HLP s'est effectué sans problème. Depuis l'origine du quai, pour prévenir un patinage lors du départ, je sable le rail, arrêt à quelques mètres de la rame. Nous profitons du stationnement pour astiquer le corps cylindrique qui a une "belle peau ", puisque la série de locomotives est de construction très récente. 


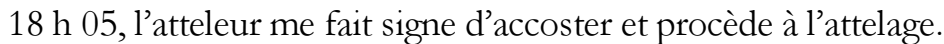
La pression de la chaudière est voisine du timbre, 20 bars, le niveau de l'eau dans le tube est à la moitié. Pour éviter une levée de soupape très bruyante, $j$ 'ouvre légèrement le régulateur BP (basse pression), ce qui permet de réduire la pression de la chaudière et de réchauffer le groupe cylindres BP, limitant ainsi lors du départ des condensations très néfastes à la bonne tenue des joints et des garnitures des tiges de pistons. Le chef de sécurité me remet le bulletin de composition de Paris à Troyes : 11 véhicules, 44 essieux, 464 tonnes ; de Troyes à Chalindrey : 7 véhicules, 28 essieux, 312 tonnes. L'essai des freins est effectué. L'heure de départ est proche, Jean s'occupe du feu, souffleur en grand, à l'aide du croc ou ringuard, il étale progressivement le feu, flanc par flanc, tout en observant la couleur de la fumée qui grise sans être noire est admise par le « caporal fumées » qui est en bout de quai.

18 h 22 c'est l'heure du départ, signal réglementaire du chef de sécurité. La chaudière à la pression voisine de 20 bars, la hauteur de l'eau dans le tube est de $4 \mathrm{~cm}$. Jean n'est pas un novice, c'est un excellent chauffeur. Vu la charge, je démarre en double expansion ou compound jouant avec le régulateur HP (haute pression). Le train s'ébranle, j’utilise par intermittences les sablières jusqu'à la hauteur de la première aiguille. Avec le stoker ou chargeur mécanique, Jean alimente modérément le foyer, porte entreouverte pour limiter les émissions de fumée. Sur la droite démarre le banlieue de 18 h 22 destination Gretz, 9 voitures remorquées par une 141 TB 400 ex 4400 Est. Pour éviter des primages ${ }^{7}$ j'ai pour habitude de ne pas brutaliser notamment lors des premiers démarrages. Le train de banlieue prend de l'avance, son démarrage est énergique. Bonnes locomotives les $141 \mathrm{~TB}$. La chaudière se maintient en pression, la pompe ACFI en service alimente la chaudière en eau. $\mathrm{A}$ hauteur du pont Riquet ( $\mathrm{Pk}$ 1), la vitesse est de $30 \mathrm{~km} / \mathrm{h}$, j'ouvre progressivement en grand le régulateur HP, marche d'admission à $40 \%$, la puissance de la P 250 se fait sentir. Le bruit caractéristique de l'échappement kylchap se fait entendre, nous circulons sur voie 1 , le banlieue sur voie 1 bis, sur voie 1 ter j'aperçois au loin l'arrière du train 25, départ $18 \mathrm{~h} 20$ pour Mézières-Charleville remorqué par $231 \mathrm{~K}$ du dépôt de Paris-la-Villette. Les voies sont équipées en BAM (block automatique mécanique Est). Nous dépassons le train de banlieue et saluons au passage l'équipe de la 141 TB. Un coup d'œil au pyromètre de la vapeur surchauffée, elle est à $280^{\circ}$ et son aiguille bien stable est un signe de non début de primage, observation très utile lors des fortes

7- Entraînements d'eau dans la vapeur qui nuisent au bon graissage des cylindres. 
admissions. La chaudière se maintient au timbre, je règle le cran d'admission en fonction de la montée en vitesse. J'aborde la gare de Pantin, la vitesse est proche de $85 \mathrm{~km} / \mathrm{h}$, je vais procéder à l'essai de frein en ligne. Ce n'est pas l'endroit idéal, mais le chef de dépôt de l'époque exige que cet essai soit effectué dans la mesure du possible avant la première bifurcation. Il est intransigeant d'où le surnom de " Médor ». Un signe à Jean, il arrête le stoker, met en action le souffleur, entrebâille la porte du foyer pour éviter la fumée. Dépression de 0,500 bar à la conduite générale, les freins répondent, desserrage. La chute de vitesse est de $15 \mathrm{~km} / \mathrm{h}$, reprise de la traction, régulateur HP ouvert en grand, cran d'admission à $35 \%, 19$ bars au manomètres $\mathrm{HP}, 6,5$ bars à la BP, aiguille du pyromètre à 325 , stable. La vitesse remonte progressivement, le kylchap rugit. Nous approchons de la gare de Noisy-le-Sec. Les deux feux blancs sur la potence m'indiquent que la direction est bonne, la vitesse est de $88 \mathrm{~km} / \mathrm{h}$, il est $18 \mathrm{~h} 32$ bon "poids ", pour $18 \mathrm{~h} 31$ passage horaire soit une minute de retard, cause de l'essai de frein non inclus dans l'horaire (9 minutes prévues de Paris à Noisy-le-Sec). La vitesse se stabilise dans la montée de Rosny, rampe de 4 pour mille, admission

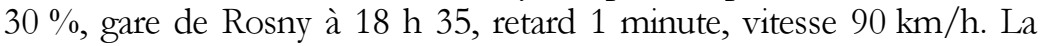
pente de 3 et 5 pour mille permet d'atteindre les $105 \mathrm{~km} / \mathrm{h}$, je réduis le cran d'admission à $20 \%$ et la pression aux cylindres HP à 6,5 bars pour obtenir une pression de 1,5 bars aux cylindres BP pour un bon maintien des grosses têtes des bielles $\mathrm{BP}$, règle impérative de conduite des $141 \mathrm{P}$. Nogent-le-Perreux et son viaduc sur la Marne, la vitesse est d'un bon $105 \mathrm{~km} / \mathrm{h}$, nous attaquons la rampe d'Émerainville 6 et 7 pour mille sur $8 \mathrm{~km}$. Régulateur ouvert en grand, cran d'admission entre 30 et $35 \%$. La vitesse décroît lentement, Villiers-sur-Marne 18 h 40, retard 1 minute, vitesse $100 \mathrm{~km} / \mathrm{h}$. La section de Nogent-le-Perreux est équipée en BAL type est (block automatique lumineux dont les particularités sont : l'allumage de signaux par approche de train, et la marche à vue sur deux cantons lors du franchissement d'un signal donnant l'indication sémaphore). Le haut de la rampe est franchie à $90 \mathrm{~km} / \mathrm{h}$, Émerainville

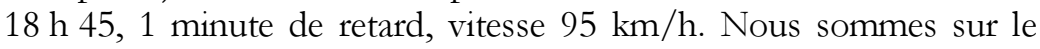
plateau de Brie, la ligne est en palier puis en pente de 3 pour mille permet d'atteindre la vitesse de $105 \mathrm{~km} / \mathrm{h}$ (vitesse limite des $141 \mathrm{P}$ ) dans la forêt d'Armainvilliers; Gretz à 18 h 52 toujours la minute de retard, à quelques secondes près, nous serons à l'heure à Verneuil. La ligne est en pente de 4 pour mille, Villepatour vitesse $100 \mathrm{~km} / \mathrm{h}$, le palier et rampe de 2 pour mille font chuter la vitesse à un bon $90 \mathrm{~km} / \mathrm{h}$ pour aborder la descente 6 pour mille d'Ozoir-le-Voulgis avec 6,5 bars à la $\mathrm{HP}$ et 1,5 bars à la BP la courbe d'Ozoir est franchie à une vitesse de $105 \mathrm{~km} / \mathrm{h}$. La ligne suit la vallée de la Marsange et franchit l’Yerres sur 
un viaduc. C'est la montée sur Verneuil-l'Étang en rampe de 4 pour mille, régulateur ouvert en grand, cran d'admission à $25 \%$ puis c'est l'arrêt de Verneuil. Réduction de la pression vapeur aux cylindres dépression de 0,5 bar à la CG (conduite générale d'air comprimé nécessaire pour le freinage) avant le signal d'entrée de Verneuil, réduction de la vapeur aux HP en fonction de la chute de vitesse. Serrage accentué à la demande par petites dépressions, fermeture du régulateur au milieu du quai, arrêt à $19 \mathrm{~h}$ 01, nous sommes à peu près à l'heure. Je profite de la minute d'arrêt pour plonger dans le mouvement BP par le tablier droit côté avant de la machine. La température des grosses têtes et petites têtes bielles BP est normale, 19 h 03 départ, retard 1 minute. La ligne est équipée en block manuel avec circuit de voie (BMCV).

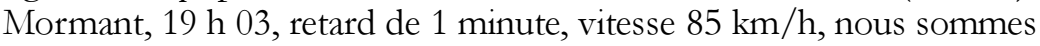
en pleine Brie avec ses champs à perte de vue. La vitesse de $105 \mathrm{~km} / \mathrm{h}$ est atteinte après le pont de la nationale 19, puis c'est l'arrêt de Nangis à 19 h 17, gain d'une minute, 19 h 19 le départ. Le démarrage de Nangis en palier est facile ; malgré la courbe suivie d'une rampe de 1 et 4 pour mille, nous atteignons le point culminant du parcours de Paris à Troyes à hauteur du disque de Maison-Rouge (altitude 138 mètres). C'est la plongée sur la vallée de la Seine par une pente de 6 pour mille sur $15 \mathrm{~km}$. Passage à Maison-Rouge à $19 \mathrm{~h} \mathrm{28}$, le palier de la gare fait chuter la vitesse à $100 \mathrm{~km} / \mathrm{h}$, le 105 est vite repris et quelque peu dépassé ce qui impose un freinage. Nous descendons la vallée du Dragon dont les sources sont captées pour alimenter en eau la ville de Paris. J'arrive dans mon pays natal : le disque de Longueville, le souterrain des Bouchots, toujours le $105 \mathrm{~km} / \mathrm{h}$, dépression de 0,5 bar à la CG peu avant le viaduc de Besnard sous lequel coule la Voulzie dont les sources sont aussi captées par la ville de Paris. Coups de sifflet au passage pour mes parents. L'arrêt de Longueville n'est pas des plus faciles, il ne faut pas s'y prendre trop tard sous peine de dépassement du quai. Longueville à l'heure 19 h 34 . Le train de Provins des CFTA (dit «le bricolo») attend ses voyageurs. Une $230 \mathrm{~B}$ manœuvre et trie les wagons pour formation au départ et assure la réserve. C'est à ce poste que j'ai fait mes débuts de chauffeur en 1947. Un regard vers le dépôt, départ 19 h 36 à l'heure. La voie en courbe est génératrice de patinages, il ne faut pas être violent. Un palier de deux kilomètres permet une montée en vitesse à $85 \mathrm{~km} / \mathrm{h}$. La ligne suit la vallée de la Voulzie. Coups d'œil au pyromètre, aiguille bien stable $350^{\circ}$, aucun signe de primage, c'est le début d'une pente de 6 pour mille, la vitesse est de $85 \mathrm{~km} / \mathrm{h}$, cran d'admission amené à $20 \%$, pression boîtes à vapeur HP 7 bars, 1,5 bars à celles des BP. Flamboin $19 \mathrm{~h}$ 41, vitesse $105 \mathrm{~km} / \mathrm{h}$. Sitôt la fin de la courbe, régulateur ouvert en grand, la ligne en faible rampe suit la vallée de la Seine, permet de 
maintenir le $105 \mathrm{~km} / \mathrm{h}$. Hermé franchit, nous roulons dans le cours de la vieille Seine, refuge des sangliers, une vraie jungle, des marécages, roseaux, buissons, de grands peupliers... Nogent-sur-Seine approche, serrage à l'entrée de la courbe précédant la gare, arrêt $19 \mathrm{~h}$ 52, à l'heure. Je vérifie la température, des bielles, des boîtes d'essieux côté gauche de la $\mathrm{P} 250$ et de son tender : rien à signaler, départ à 19 h 54. Ligne en rampe de 4 pour mille pour franchir la Seine sur le pont de Bernière (lieu de prédilection pour la pêche de quelques mécaniciens de Paris-La Villette), suivi d'une pente de 4 pour mille qui permet d'atteindre la vitesse de $105 \mathrm{~km} / \mathrm{h}$. Nous suivons le canal latéral de la Seine, la petite halte de Marnay. Gare de Pont-de-Seine 20 h 03, halte de Crancey, le profil un peu difficile m'oblige à augmenter l'admission, la chaudière se maintient au timbre, le niveau d'eau dans le tube est soutenu. C'est l'arrêt de Romilly, en ligne droite, quai long mais à droite, ne pose pas de problème, serrage peu avant le pont précédant la gare. Romilly $20 \mathrm{~h} \mathrm{10}$. Dès l'arrêt, je profite du quai à droite pour vérification de la température des bielles et boîtes d'essieux côté droit tandis que Jean plonge dans le mouvement intérieur pour contrôler la température des grosses têtes et petites têtes bielles BP. Tous ces ensembles ont une température normale ${ }^{8} .20 \mathrm{~h} 12$ départ: mise en vitesse facilitée par une courte pente suivie d'un palier jusque Maizières-la-Grande-Paroisse, passage $95 \mathrm{~km} / \mathrm{h}$.

La ligne remonte la vallée de la Seine par une succession de rampes de 2 à 3 pour mille entrecoupées de faibles et courtes pentes et paliers. De Romilly à Troyes, l'horaire est tendu, il faut tenir le $105 \mathrm{~km} / \mathrm{h}$, atteint entre Châtres et Mesgrigny-Mery.

La P 250 « gaze bien », elle tient le timbre. La ligne est assez rectiligne, haltes, petites gares se succèdent : Vallant-Saint-Georges, SaintMesmin, Savières, Payns, Saint-Lyé, puis Barberey située à l'entrée du triage de la Chapelle-Saint-Luc à droite.

Sur la gauche, le dépôt de Troyes, avec sa rotonde typique de la région est ; sur une voie de garage des locomotives toutes rouillées, 230 $\mathrm{A}, \mathrm{B}, \mathrm{K}, 231 \mathrm{C}$ ex PO (dont la C 401 ex $3566 \mathrm{PO}$ première transformée en 1929) attendent l'heure de la démolition, c'est déjà le déclin de la vapeur. Troyes-Preize - TIV 30 (tableau indicateur de vitesse), à l'entrée de la courbe à droite, admission réduite aux boites à vapeur, serrage, respect de la zone à $30 \mathrm{~km} / \mathrm{h}$ qui est la vitesse de circulation de la gare

8- En cas de températures anormales d'un organe à Romilly, un avis au régulateur EX (Exploitation) permet de faire sortir la machine de réserve secours de Troyes pour échange de locomotives en gare de Troyes sans aucune perte de temps. 
de Troyes, gare d'arrêt général imposant la marche à vue. Il faut arrêter à la grue pour prise d'eau. Je ne suis pas un artiste au frein automatique pour ajuster cet arrêt : l'orifice de remplissage des tenders $34 \mathrm{P}$ étant à l'arrière de ceux-ci. De plus, la rame du 409 est composée de matériels français, équipés de triple valve, et de matériels ex DR équipés de distributeurs HIk (Hidelbrank Knorr) et kk (Kunz-Knorr) ce qui rend la manœuvre du frein délicate pour de tels arrêts. Sur la fin du parcours, j'utilise judicieusement le frein direct de la machine pour obtenir l'arrêt au point voulu sans réaction de la rame.

Je m'occupe du complément d'eau pendant que Jean plonge de nouveau dans le mouvement BP pour faire un complément d'huile aux grosses têtes de bielles BP (basse pression). L'arrêt de Troyes 19 h 39 19 h 46 soit 7 minutes me permet de vérifier le débit des graisseurs des bielles HP (haute pression), de graisser quelques articulations du petit mouvement. Le tender est complété en eau, le chef de sécurité m'informe que les quatre voitures de queue sont dételées. Jean à l'aide du grand croc gratte la grille, met des mâchefers sur les flancs du foyer.

19 h 46 : signal de départ, démarrage sans difficulté, 7 voitures, 324 tonnes ne présentent pas de problèmes de mise en vitesse. Le $100 \mathrm{~km} / \mathrm{h}$ est atteint à la bifurcation de Saint-Julien, la Seine est franchie après cette bifurcation. Le profil de la ligne est une succession de rampes de 2 à 5 pour mille coupées de courts paliers et pentes, la marche est à $20 \%$ d'admission : il faut « jouer» du régulateur pour soutenir une vitesse de 100 à $105 \mathrm{~km} / \mathrm{h}$ exigée pour tenir l'horaire. La ligne est équipée en block automatique mécanique type est de Troyes à Culmont-Chalindrey. Suite aux accidents de Bollène et Nozières sur le Sud-Est, la non répétition des signaux, même à voie libre, doit être impérativement signalée. Sur ce parcours, selon le profil de la ligne, les avertissements sont groupés ou non avec les sémaphores, d'où une certaine difficulté la nuit et surtout par temps de brouillard et de neige pour repérer ces signaux.

Nous remontons la vallée de la Barse, gare de Lusigny à 20 h 58 avec une minute d'avance. À Montieramey, nous passons sur le pont du canal qui alimente le lac de la forêt d'Orient.

La petite halte de la Villeneuve-au-Chêne suivie d'une petite faïencerie éclairée la nuit indique l'approche de Vendeuvre où le 409 s'arrête : viaduc peu avant la gare et c'est l'arrêt à Vendeuvre-sur-Barse 21 h 11 $21 \mathrm{~h} \mathrm{12}$. Dès la sortie de la gare la rampe est de 5 pour mille sur $6 \mathrm{~km}$. La 141 P 250 avec ses 324 tonnes grimpe allègrement cette rampe : vitesse au sommet $85 \mathrm{~km} / \mathrm{h}$ à Vauchonvilliers-Maison-des-Champs ; admission $20 \%, 7$ bars aux HP, 1,5 bar au BP pour descendre Jessains. 
La pente est de 6 pour mille sur $8 \mathrm{~km}$ pour suivre la vallée de l'Aube jusqu'à Clairvaux. La courbe et le palier de la gare de Jessains font chuter de quelques $\mathrm{km} / \mathrm{h}$ la vitesse ce qui évite d'effectuer un freinage dans les derniers kilomètres de la pente.

Arrêt à Bar-sur-Aube, 21 h 29 - 21 h 31, et dès le départ la ligne est en rampe de 5 pour mille suivie d'un palier où la vitesse de $100 \mathrm{~km} / \mathrm{h}$ est atteinte sans peine.

De Bar-sur-Aube à Chaumont c'est une suite de rampes continues de 2, 3, 5 et 6 pour mille entrecoupées de courts paliers sur une distance de $41 \mathrm{~km}$.

Cran de marche à $20 \%$, régulateur ouvert en grand, la P 250 malgré les rampes de 2 et 3 pour mille soutient les $100 \mathrm{~km} / \mathrm{h}$, la halte de Bayel avec sa cristallerie renommée, Clairvaux à 21 h 40, Maranville, la rampe s'accentue, 5 et 6 pour mille, continue jusqu'au viaduc de Chaumont. J'augmente quelque peu l'admission à $25 \%$, la 250 s'accommode bien de ces rampes et soutient une vitesse comprise entre 85 et $90 \mathrm{~km} / \mathrm{h}$ suffisante pour réaliser l'horaire. Passage à Bricon, 21 h 52 (1 minute d'avance). Les voies bis sur la gauche ne sont pas déposées de Bricon à Villiers-le-Sec, et servent de garage à des locomotives en attente de démolition. Le palier en gare de Villiers-le-Sec permet d'atteindre les $100 \mathrm{~km} / \mathrm{h}$. La voie 1 ligne 10 (Laon à Chaumont) nous rejoint peu avant le viaduc de Chaumont, viaduc d'une longueur de 606 mètres pour une hauteur de 52 mètres. C'est l'arrivée de Chaumont, ralentissement $30 \mathrm{~km} / \mathrm{h}$ présenté, serrage sur le viaduc, rappel de ralentissement 30 pour franchir l'aiguille qui donne accès à la voie 1 bis ou le 409 est reçu pour desserte de la Poste. Chaumont à 22 h 04 (avance une minute), contrôle de température des bielles HP et BP et boites de roues, contrôle plus moral que nécessaire, il n'y a plus que $46 \mathrm{~km}$ à parcourir.

Départ à $22 \mathrm{~h} \mathrm{10}$, la ligne est en pente de 6 pour mille pour rejoindre la vallée de la Marne suivie pendant $4 \mathrm{~km}$ environ après la gare de Langres, près de sa source. Passage devant le dépôt de Chaumont à $75 \mathrm{~km} / \mathrm{h}$, je réduis l'admission à $20 \%$ ainsi que la pression aux HP et BP pour atteindre les $105 \mathrm{~km} / \mathrm{h}$ où la ligne rejoint le canal latéral à la Marne peu avant Luzy. De cette gare à Langres, la marche tendue exige de tenir une vitesse de $105 \mathrm{~km} / \mathrm{h}$. La ligne est en rampe continue de 2, 3, 5 et 6 pour mille. Foulain à $22 \mathrm{~h} \mathrm{19}$, régulateur ouvert ou grand, cran d'admission à $25 \%$. Les souterrains de Marnay et La Pommeraie sont à ce jour fermés et remplacés par deux nouveaux souterrains. 
Rolampont $22 \mathrm{~h} 27$ à l'heure, je repousse le régulateur et réduis l'admission à $20 \%$ sur une pente de 3 pour mille sur un kilomètre environ, ce qui a pour conséquence un dépassement de vitesse, il faut bien connaitre cette partie de ligne car, dès la fin de la pente, pour maintenir les $105 \mathrm{~km} / \mathrm{h}$ il faut admettre une pression maximum et augmenter le cran d'admission. Nous franchissons la halte de Humes, la ligne suit le canal latéral de la Marne, dans une courbe à gauche apparait le triage de Jorquenay, la gare de Langres n'est plus loin, arrêt classique avec une pression de 1 bar à 1,5 bar aux cylindres BP jusque dans les derniers mètres de l'arrêt.

Langres, 22 h 36 départ à 22 h 38 . Plus que 11 kilomètres. Il faut franchir la ligne de partage des eaux de la Manche et de la Méditerranée par une rampe de 3 et 6 pour mille suivie d'une pente de 7 pour mille jusque en gare de Culmont-Chalindrey. La P 250 toujours au timbre avale cette rampe sans problème. Sur la droite, nous sommes près des sources de la Marne. Le sommet de la rampe est franchi à $95 \mathrm{~km} / \mathrm{h}$. Je repousse le régulateur, la ligne est en pente, Jean met le souffleur pour éviter les retours de flammes lors du passage dans le souterrain de Culmont long de 1325 mètres. Dès la sortie du souterrain, apparaissent en contrebas les lumières de la gare de Culmont. Vitesse de $105 \mathrm{~km} / \mathrm{h}$, successions de signaux mécaniques, premier avertissement ouvert, TIV 80 effacé, deuxième avertissement présenté, j’effectue le serrage des freins avant de franchir cet avertissement (principe que $j$ 'ai toujours appliqué lors des circulations près de la vitesse limite), troisième avertissement présenté. Nous abordons le quai où stationne sur voie 2 l'YM (Metz-Marseille) remorqué par une $231 \mathrm{G}$ ou K de Nancy, arrêt devant le passage planchéié. Il est $22 \mathrm{~h} 49$, à l'heure. Un agent de la gare nous attend pour couper la machine sans oublier la mention habituelle : « appuie un peu pour couper. » Le chef de sécurité demande le bulletin de composition. En bout de quai, sur potence, le panneau lumineux : trois feux jaunes (avertissement, rappel de ralentissement 30) tableau D nous indiquent que nous allons au dépôt. Le chef de sécurité fait signe d'avancer (sécurité du passage planchéié). Sur l'YM, repris par l'arrière, une $141 \mathrm{P}$ des « Sorciers » (agents de Chalindrey) est en tête et est prête à s'élancer sur Dijon. Nous rentrons au dépôt. Une 141 P de Noisy est au carré de sortie, pour assurer le 478 de Culmont à Langres (3 voitures du 409) puis le 4050 pour Paris-Est. Petit coup de sifflet au passage et bonne nuit. Arrêt sur fosse spécialisée pour extraction (TIA). Visite sur fosse de rentrée ; pendant que j'effectue la visite de l'engin, Jean décrasse les deux plans grille avant du foyer et envoie sur chaque plan de grille une vingtaine de pelles de charbon pour confection du talon (maintien de la 
machine au feu). Après visite, la 250 est bonne pour assurer le 410, passage au toboggan pour chargement : $4800 \mathrm{~kg}$. Mise en place pour remplir le tender en eau. Le surveillant de dépôt prend possession de la machine et s'informe s'il y a des réparations à effectuer pour une mise éventuelle en rotonde. Passage au sous-chef de dépôt qui prend attachement de la machine et de l'équipe. C'est la direction du foyer avec nos affaires personnelles. Au réfectoire bien occupé, les équipes arrivantes se restaurent, ce que nous allons faire après nous être lavés et avoir changé de tenue. Les équipes partantes boivent le café, sans oublier les acharnés du tarot. La nuit ne sera pas longue, nous repartirons au 410,

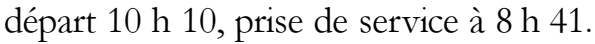

Sous ma responsabilité, Jean assurera la conduite du 410, c'était la méthode de formation du temps de la vapeur.

À Longueville, Papa Lécheneau, mécanicien retraité, sera en tête du quai avec deux bons casses-croûte, fruits et bouteille de Vitteloise qui seront avalés lors de la montée de la rampe de Maison-Rouge. 